\title{
Static and Dynamic Structure Characteristics of a Trust Network and Formation of User Trust in an Online Society
}

\author{
Ying Li, Hongduo Cao*, Ying Zhang \\ Business School, Sun Yat-sen University, Guangzhou, China \\ Email: *caohd@mail.sysu.edu.cn
}

How to cite this paper: Li, Y., Cao, H.D. and Zhang, Y. (2018) Static and Dynamic Structure Characteristics of a Trust Network and Formation of User Trust in an Online Society. Social Networking, 7, 197219. https://doi.org/10.4236/sn.2018.74016

Received: March 27, 2018

Accepted: August 12, 2018

Published: August 15, 2018

Copyright (C) 2018 by authors and Scientific Research Publishing Inc. This work is licensed under the Creative Commons Attribution International License (CC BY 4.0).

http://creativecommons.org/licenses/by/4.0/

\section{(c) (i) Open Access}

\begin{abstract}
This study investigated the characteristics and formation of the online social trust network of Epinions.com, a general consumer review site. An analysis of the static structure of this social trust network revealed a high clustering coefficient, short average path length, and power-law degree distribution; it is therefore a small-world and scale-free trust network. The dynamic evolutionary characteristics of the online social network (OSN) were also examined. The results showed that the scale of the network followed a sigmoidal curve; the average degree of the network was nonconstant and changed into a bell-shaped distribution; the density of the network decreased and subsequently stabilized; and user trust diffusion in the network conformed to the Bass model. Finally, the formation of trust within the network was researched at the overall network (macro) and individual user (micro) levels. Compared with their accumulated contribution and reputation, user activeness had a larger effect on trust formation in OSNs, indicating a "diminishing returns" phenomenon. This phenomenon contrasts with the Matthew effect (i.e., the more reputation a person has, the more likely he or she is to be trusted) in real-world social networks.
\end{abstract}

\section{Keywords}

Trust Network, Static Structure, Dynamic Evolution, Trust Formation, Diminishing Returns

\section{Introduction}

Trust is fundamental in constructing a society. Strong social trust facilitates the operation of social capital. The formation of trust within a society involves nu- 
merous factors such as social operating mechanisms (e.g., social institutions and socio-cultural environments) and the characteristics and behaviors of individual members of that society.

Trust is a subjective belief that can be externalized within an online social network (OSN) as a network connection. Trust can therefore be directly observed in OSNs. Moreover, trust between social network members may be associated with each member's social status and behavior.

The construction of a social network depends on the formation of nodes and edges, the latter of which has received widespread academic attention. Generally, connections between network nodes are formed by the static characteristics of the nodes, such as reputation and the number of existing links. However, such characteristics are inadequate for explaining the network construction process. In addition, the meaning of a microanalysis of trust formation differs from that of a macroanalysis, and many previous studies have focused on only one of these approaches.

In the present study, OSN data were employed to examine network connections as external characteristics of trust. The overall change in the network was identified to investigate trust formation within the OSN at the macro- and micro-levels. At the macro-level, the effects of the static structure and dynamic evolution of the network on the increase of the number of its edges were measured. At the micro level, panel data were used to evaluate the effects of individual behaviors on trust formation within the OSN. The micro- and macroanalyses of the effects of the static and dynamic characteristics of the network and its nodes on trust formation reached an agreement: user activeness exerted a larger effect on trust formation than did their accumulated contribution and reputation. A diminishing returns phenomenon was observed in the relationship between user activeness and trust formation in the examined OSN, which is in contrast to the Matthew effect (i.e., the more reputation a person has, the more likely he or she is to be trusted) in real-world social networks.

\section{Literature Review}

A social network is a typical complex network comprising a set of relationships between individuals or groups. The term is used to describe such relationships within a given context. Within a social network, a node represents an individual and a connection defines a particular relationship between two individuals (e.g., friendship, kinship, work relationship, research collaboration, or business relationship).

Previous studies have investigated such topological characteristics of social networks as the orientation (directed versus undirected), the total number of nodes and edges, the average degree and path length, and the clustering coefficient. Many social networks exhibit a power-law degree distribution.

The growing prevalence of computers and the continued development of the Internet enable increasingly more people to connect with each other in the vir- 
tual world. Computer networks have reshaped social relations and interactions, creating a social network known as an OSN. The advent of OSNs coincided almost exactly with that of the Internet; examples of such networks include e-mail services, message boards, dating websites, online (virtual) communities, and media websites. OSNs reflect yet differ from actual interpersonal relationships. Face-to-face social interactions entail considerable time and effort invested in maintaining relationships, thus limiting the number of personal connections a person can make. By contrast, the Internet enables people to acquaint themselves with each other within a shorter time frame. Thus, the efficiency of networking defines the major structural and functional differences between faceto-face and Internet-based interactions.

OSNs have played a vital role in human life and have been extensively studied. Mainstream research has focused on OSN structures and evolutionary characteristics as well as human behavior within such networks. OSNs composed of friend connections include Club Nexus [1] [2], Facebook [3], the Renren Network [4], MySpace [5], Mixi [6] [7], Cyworld [5], iWiW [8], Orkut [5], and pussokram.com [9] [10] [11]. OSNs composed of connections between users include the virtual community at Stanford and the virtual student community at the Massachusetts Institute of Technology [1] [12] [13], Yahoo! 360 [14], and LiveJournal [15]. OSNs composed of communication between users include a bulletin board system at the Korea Advanced Institute of Science and Technology [16], Gnutella [17], MSN [18] [19], nioki.com [20], and the Java Forum [21]. Past studies have shown that OSNs and real-world social networks have small worldness, although only the former exhibits a power-law degree distribution.

Many researchers have discussed the OSN evolutionary characteristics. For example, Holme studied pussokram.com (a Swedish online dating site) and Kumar et al. investigated the structure and evolution of Yahoo! 360 [11] [14]. The relationship between time and the network average degree was shown to be nonmonotonic and to comprise three stages: the network average degree begins with a gradual increase to the peak, declines gradually, and then steadily increases [1] [14]. Mislove et al. and Leskovec et al. have explored microscopic node behavior within Flickr, del.icio.us, and Yahoo! Answers [19] [22]. In addition, the number of users (or nodes) for Cyworld [23], MySpace [24], and Wealink.com [25] exhibited sigmoidal curves-that is, rising slowly at the initial stage, growing exponentially at the critical mass point to the peak, and finally stabilizing.

Sigmoidal curves were proposed by Rogers in his 1962 publication: Diffusion of Innovations. According to Rogers, the rate of diffusion of an innovation grows slowly initially but increases markedly when a critical mass of adopters is achieved [26]. This increase continues until most potential adopters in a diffusion system adopt the innovation, after which the diffusion rate drops to the saturation point. Thus, the number of innovation adopters relative to time exhibits a sigmoidal curve. Based on Rogers' work, Bass proposed the Bass model, which 
is crucial in research on diffusion models [27].

Trust has profound implications for actual and virtual social networks. Blau regarded trust as an element of maintaining social relations, arguing that any social relations cannot be maintained without trust. Thus, trust is indispensable to the formation of relations in social networks. The same applies to OSNs; without trust, any relations formed in such networks cease to exist.

Numerous scholars have studied the formation of trust in actual social networks. Zucker took a systematic approach to the subject, proposing three modes of trust formation: 1) process-based trust, based on expected or past exchange (e.g., reputation); 2) characteristic-based trust, based on shared social characteristics such as family background, ethnicity, and beliefs (the more similar these characteristics are, the stronger the trust is); and 3) institutional-based trust, based on formal societal structures such as professional credentialing, bureaucratic organizations, intermediary mechanisms, and laws.

The trust formation mechanism for OSNs, because of the virtuality of such networks, may differ from that for real-world social networks. Drawing on views of Zucker and Whitley regarding trust formation, Bai proposed three formation mechanisms for online interpersonal trust: presumed trust, trust based on online reputation, and trust based on subjective judgment [28]. By examining the characteristics of cyberspaces and virtual interpersonal relationships, Huang found that such relationships are based on partial interpersonal interactions and that judgments made by participants in online interactions are instrumental in whether trust exists within the relationships [29]. Based on an OSN established with trust, the present study defined each edge of the network as a formation of trust. Accordingly, this OSN was considered an online trust network.

\section{Data and Sample Selection}

\subsection{Data Description}

Inaccuracies, subjectivity, and limited sample sizes are some of the limitations that have been identified in many previous studies on real-world social networks. For example, Milgram obtained data through questionnaires and interviews, although both research methods were time-consuming and the data were subject to the personal opinions or biases of individual respondents in this study [30]. By contrast, data collected from OSNs are free from subjectivity. Thus, studying OSNs can improve clarify all issues related to real-world social networks.

Founded in May 1999, Epinions.com was a general consumer review site owned by eBay. The website provided a free and open platform where reviewers can share their opinions about the advantages and disadvantages of products to earn recognition and trust. It also provided exhaustive product information and the most objective purchase recommendations possible to help shoppers decide on a purchase.

Epinions.com attached considerable importance to trust. Two features explain 
how Epinions.com enabled shoppers to find trustworthy reviewers. One is high transparency; the website displayed every reviewer's home page, review list, review ratings, network of users trusted by the reviewer, and follower list (list of users who trust the reviewer). The other is the web of trust (based on word-ofmouth marketing), which is a list of trusted reviewers who provided purchase recommendations that other members found trustworthy or they had shared interests with that member. Members were also able to block reviewers. Epinions.com delivered reviews of reviewers to members who trusted them, but not those of reviewers who were blocked.

The home page of a reviewer (referred to as User A) would display the date of registration, location, the total number of reviews, the total number of views by members, the total number of views by members and nonmembers, title, and a personal summary. Epinions.com users were able to obtain the title of Category Lead, Top Reviewer, or Advisor. Users who frequently published high-quality product reviews had the potential to be recognized as a Category Lead or Top Reviewer, whereas those who frequently rated reviews had the potential to have been recognized as Advisors. These three titles lasted only for a certain period because Epinions.com evaluated user statuses periodically.

The web of trust, follower list, and review list of User A were also accessible on this OSN. The web of trust would provide the following categories of information about users trusted by User A: name, personal summary, location, and date of addition. Likewise, a follower list would display the names, personal summaries, and locations of users who trust User A, and dates of adding the user to their webs of trust. The review list of User A included the publication date and heading of each review, product name, product category, the user's rating score on each reviewed product, and review rating score. The names and titles of review raters, as well as their rating scores and dates of rating, were also accessible through the review list.

\subsection{Sample Selection}

\subsubsection{Sample Description}

User data were collected from Epinions.com because the information of its users is accessible and exhaustive. Given the sheer volume of Epinions.com user data, a snowball sampling approach was adopted to obtain the data.

Snowball sampling is extensively applied in OSN research. Through this method, a social relation is used as a relational chain for the construction of a sample population. Thus, a snowball sample builds around the relations of the population as the starting point around the relations ${ }^{1}$.

An Epinions.com Top Reviewer was selected as the starting point from which snowball sampling was performed to retrieve all the users who trusted (trust relation) and were trusted by (follower relation) the reviewer and those who trusted and were trusted by the users related to the reviewer, until the sample size stopped increasing. The main pages, webs of trust, follower lists, review lists, http://www.ndeehina.eom.en/nde.1043.html. 
and review scores of 91,338 users, which amounted to 86 GB of data, were obtained. Among these Epinions.com users, active ones constituted the largest user group on the website.

\subsubsection{Data Analysis}

Pajek was employed to analyze the dynamic structure of the OSN of Epinions.com because of the sheer number of nodes in the network. Pajek operates on Windows and is arguably the most effective tool for analyzing complex nonlinear networks. It enables the analysis and visualization of networks comprising thousands or even millions of nodes. MATLAB and SPSS were also applied in the estimation, analysis, statistics and goodness-of-fit test of the data.

\section{Topology and Evolution of the Social Trust Network of Epinions.com}

\subsection{The Static Structure}

In this section, the static structure of the trust network of Epinions.com was examined on the basis of the degree distribution, average path, and cluster coefficient of the network.

\subsubsection{Degree Distribution}

The trust and follower relations of the 91,338 Epinions.com users were depicted by a directed graph, whose out-degree denoted the trust relation and in-degree the follower relation. A trust network dated August 31st of 2010 was constructed from the trust and follower lists of the users. The in-degree, out-degree, and total-degree distributions of the users' trust and follower relations were all generated using Pajek (Figures 1-3). All three distributions followed a power law distribution, indicating that the network was a scale-free network. Generalized least squares was used to estimate the exponents of these distributions (out-degree $\gamma$ $=1.69$, in-degree $\gamma=1.46$, and total-degree $\gamma=1.51)$. Maximum-likelihood estimation was also used to estimate the exponents of these distributions (outdegree $\gamma=1.36$, in-degree $\gamma=1.38$, and total-degree $\gamma=1.33$ ).

\subsubsection{Average Path and Diameter}

The average path length and diameter of the trust network were 4.74 and 16, respectively. The trust network had directedness; thus, there were nonexistent links between some node pairs. In total, 4,644,430,478 inaccessible node pairs were identified in the network, accounting for $55.67 \%$ of the total number of nodes. Figure 4 presents the shortest-path distribution of the network, which is a critical global geometric quantity of a complex network; in this distribution, $P(1)$ is a path with a length of 1 . Some asymmetric unimodal functions sufficiently fitted path length distributions. Typically, the Levenberg-Marquardt method is adopted to fit the trial function $P(l)=a l \mathrm{e}^{-b l^{2}+c l}$, where $l$ is the shortest path length, $P(I)$ is the corresponding probability for 1 [31] [32]; and a, b, and c are fitting parameters. As shown in Figure 4, the data point corresponds with 


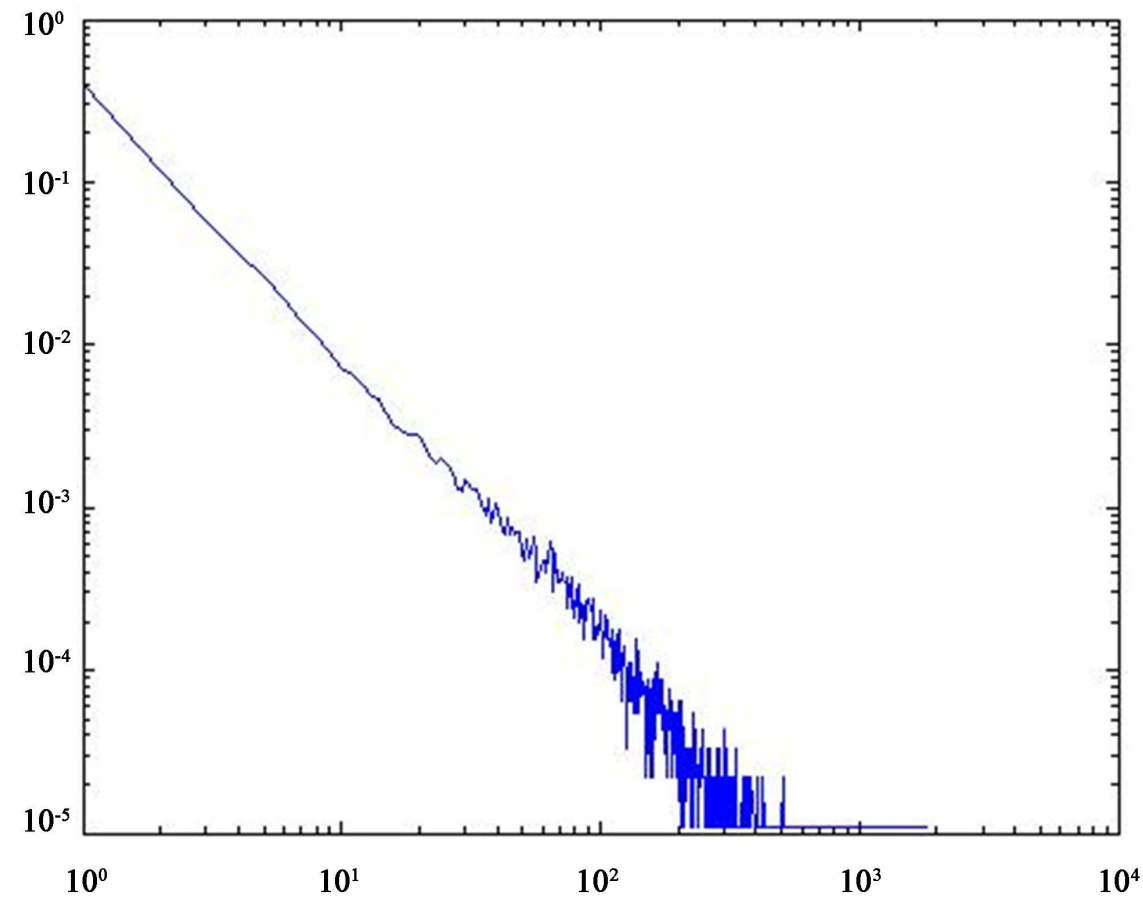

Figure 1. Out-degree distribution.

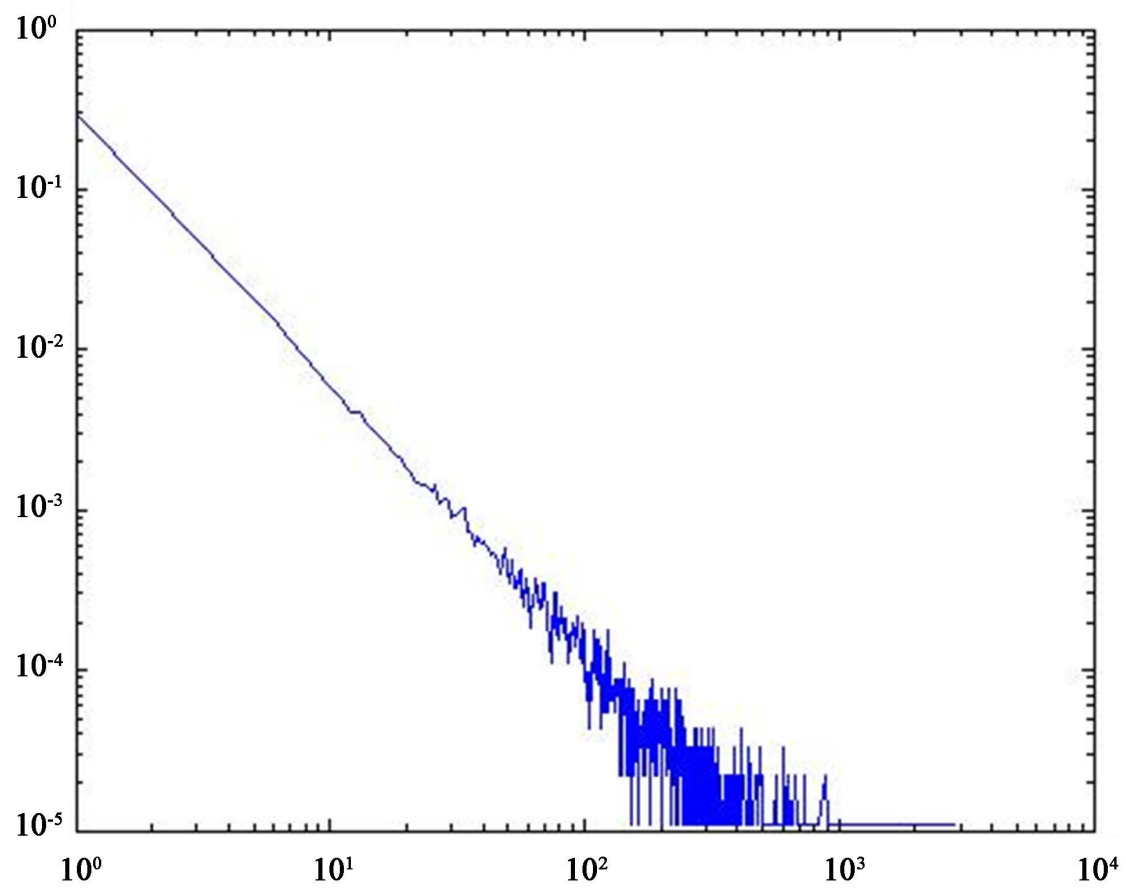

Figure 2. In-degree distribution.

the aforementioned function $(a=7.605 \mathrm{e}-006, b=0.4783, c=4.228$, and goodness of fit $=0.9978)$.

\subsubsection{Clustering Coefficient}

After the repetitive edges of the undirected portion of the trust network were 


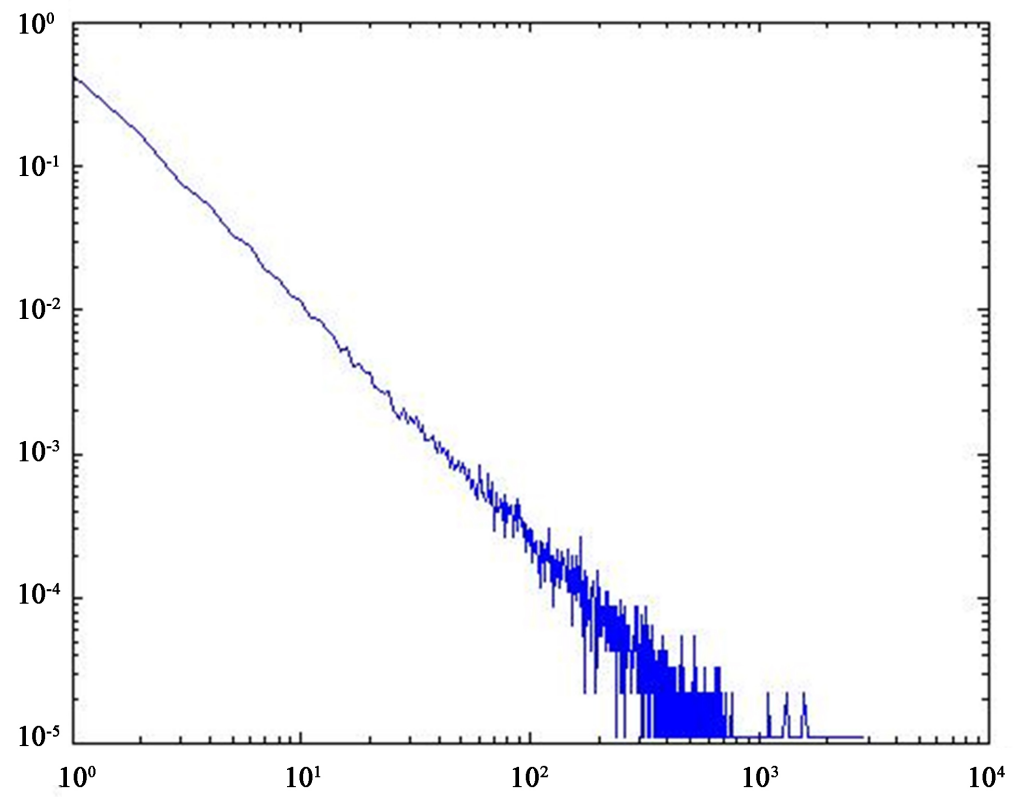

Figure 3. Total-degree distribution.

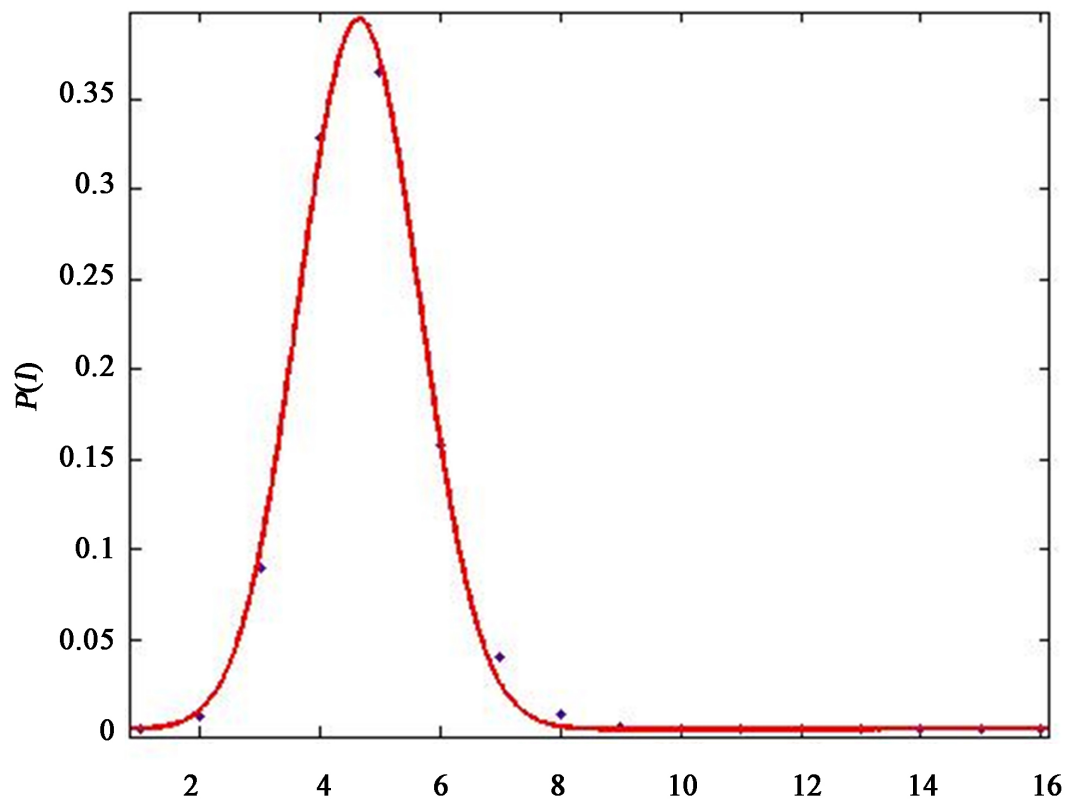

Figure 4. Shortest-path distribution.

eliminated, the average clustering coefficient of the network was 0.2643 . Compared with random networks of equivalent size, whose average cluttering coefficient is only $1.144 \times 10^{-4}$, this trust network was highly clustered.

\subsection{Dynamic Evolution}

In this section, the changes of the scale, density, and average degree of the trust network in relation to time are examined. The scale of the network was meas- 
ured according to the number of nodes and edges in the network.

\subsubsection{Growth Pattern of the Number of Nodes}

The number of users of Cyworld, MySpace and Wealink.com exhibits sigmoidal curves, corresponding with Rogers' diffusion of innovation, which describes the diffusion of a product, service, behavior, or concept within a social system [23] [24] [25]. According to the theory, the number of network nodes increases slowly at the initial stage, but then grows exponentially at the critical mass point; upon peaking, the number of network nodes stabilizes. Therefore, we referred to the registration for Epinions.com as the adoption of its service and used the Bass model to estimate the total number of nodes in the trust network of the website (based on the registration dates of the 91,338 users) as of August 31, 2010. The total number of nodes in the trust network since the website was founded exhibited a sigmoidal curve of cumulative growth (Figure 5 and Figure 6). The growth curve of the total number of nodes resembles a logistic growth curve; both are bell-shaped.

\subsubsection{Growth Pattern of the Number of Edges}

Because of the lack of time-series data on users who joined the trust network before January 11,2001, only the growth in the number of nodes in the network after that date was observed. This growth passed the slow stage and transitioned to the steady stage from the exponential growth stage. Thus, the sigmoidal cumulative growth curve and bell-shaped growth curve for the number of edges in the trust network were only partially obtained (Figure 7 and Figure 8).

\subsubsection{Evolution Pattern of the Average Degree and Density}

Different evolutionary patterns of network density have been empirically identified in recent studies. Leskovec et al. found that the average degrees of some

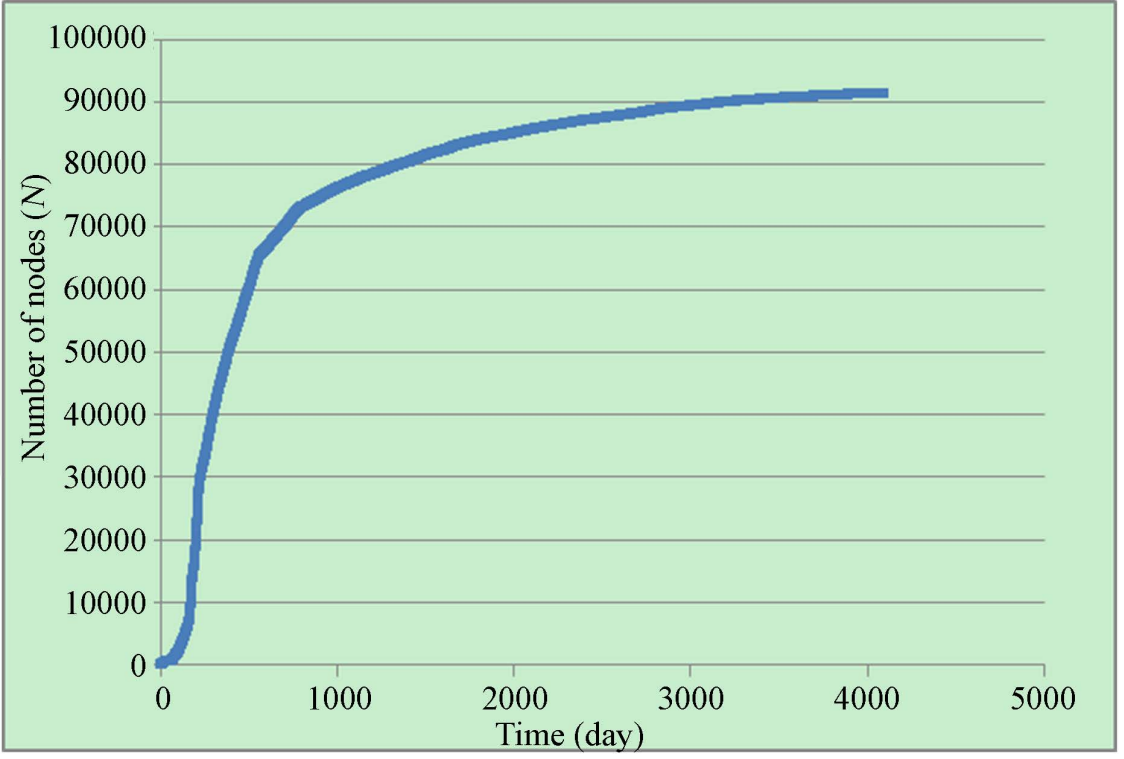

Figure 5. Cumulative growth curve of the number of nodes $(\mathrm{N})$ in the trust network. 


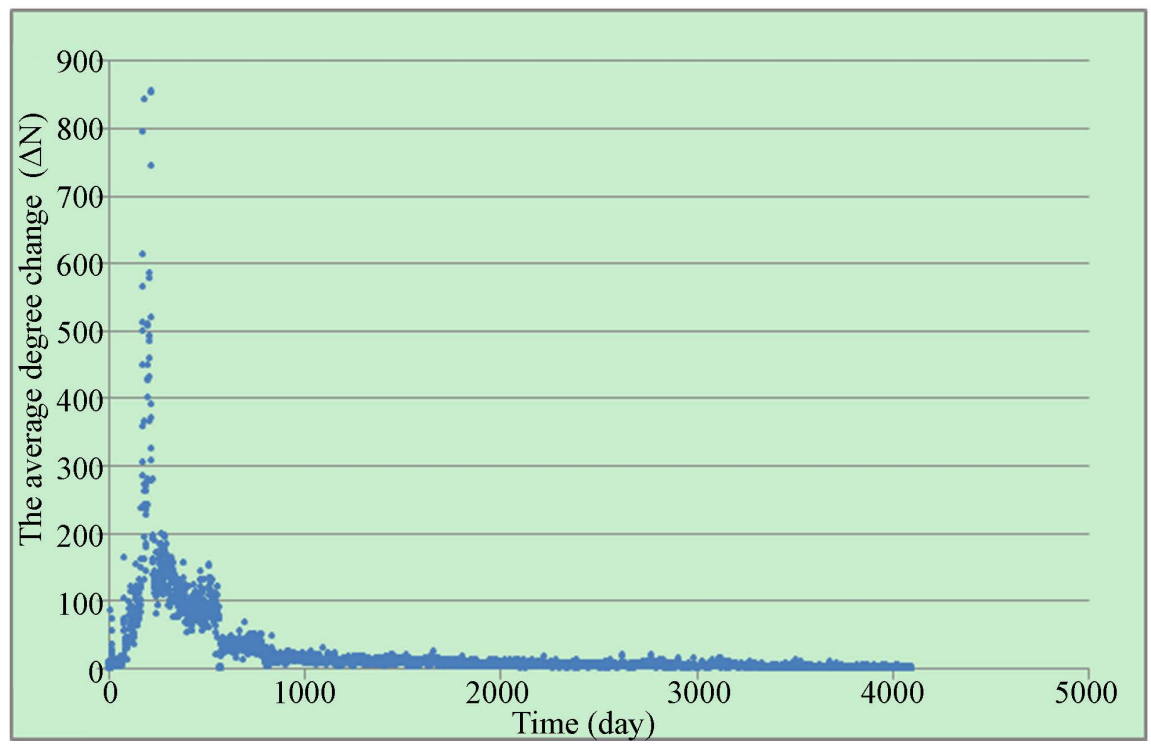

Figure 6. Growth curve of the number of nodes $(\mathrm{N})$ in the trust network.

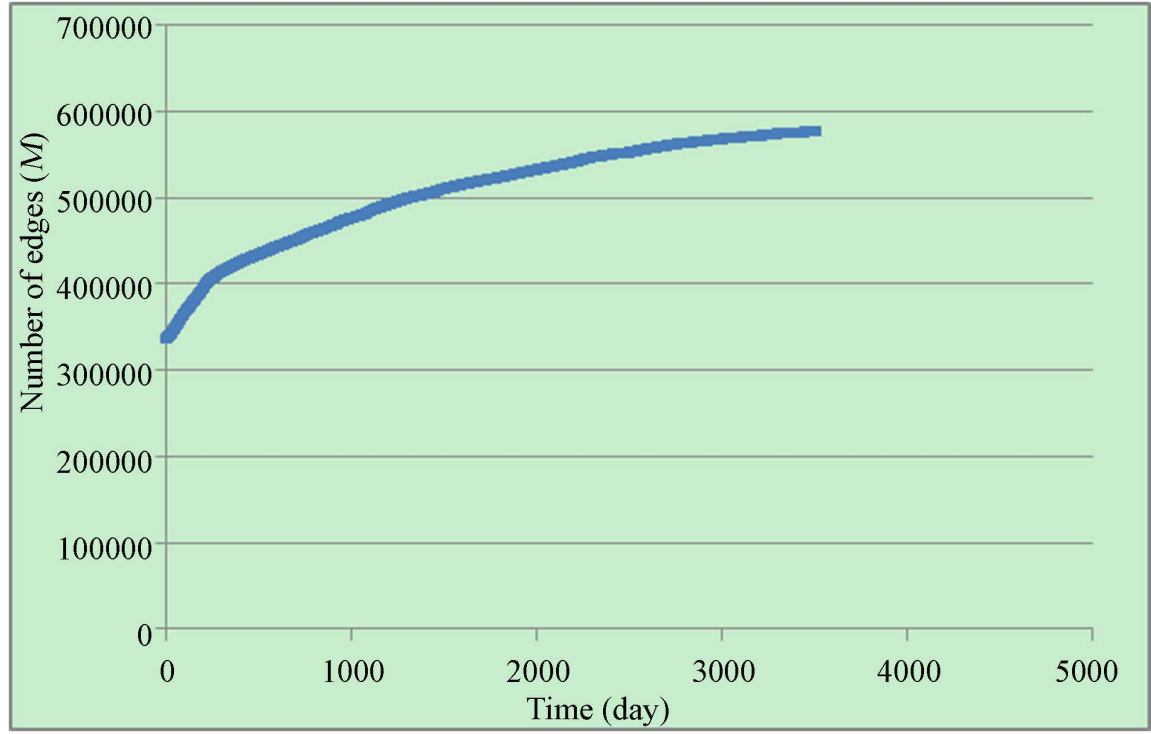

Figure 7. Cumulative growth curve of the number of edges $(\mathrm{M})$ in the trust network.

citation networks, autonomous system-level Internet, and E-mail networks increased ultralinearly with time and exhibited power-law function forms in $\log -\log$ graphs [19]. Kumar et al. found that the average degrees of Yahoo! $360^{\circ}$ and Flickr increased nonmonotonically with time, namely, increasing gradually to the peak, declining gradually to the minimum, and stabilizing thereafter [14]. $\mathrm{Hu}$ reported similar findings for Wealink.com [25].

In the present study, the average degree $(\bar{D})$ and change in the average degree $(\Delta D)$ of the trust network of Epinions.com were measured as in Figure 9 and Figure 10. The average degree of the trust network showed a nonmonotonic increase resembling a bell-shaped increase comprising three stages. It accelerated with time at the first stage, declined at the second stage, and stabilized at 6.13 at 


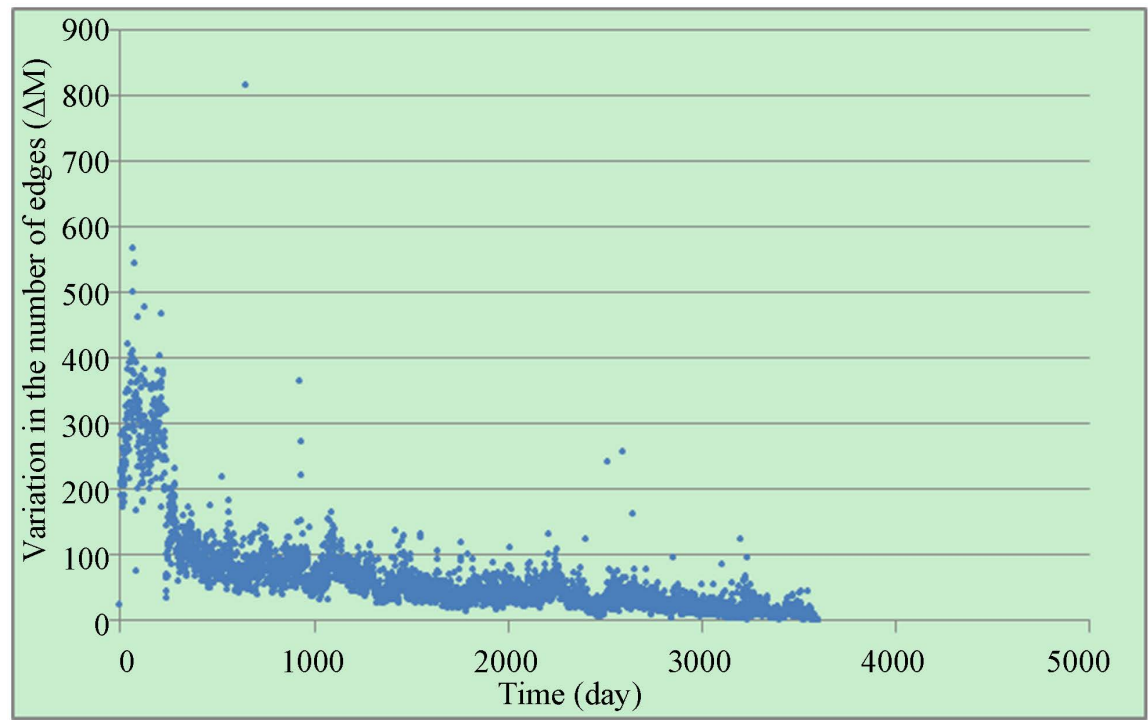

Figure 8. Growth curve of the number of edges (M) in the trust network.

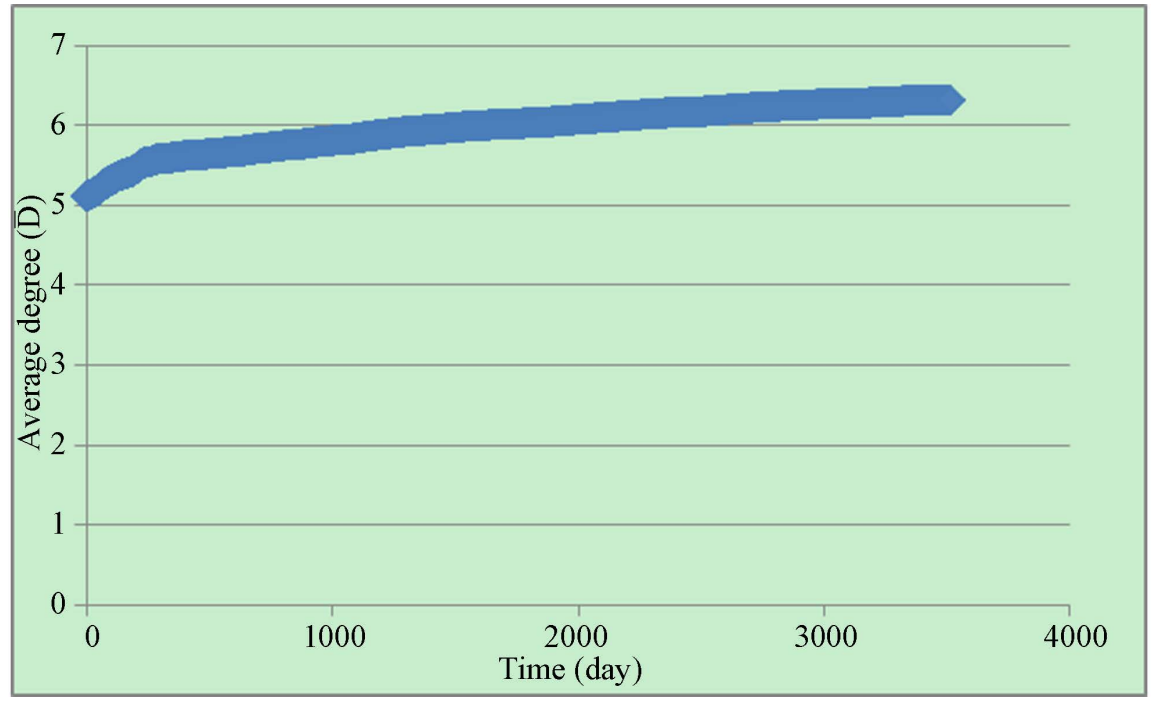

Figure 9. Average degree $(\bar{D})$ of the network in relation to time.

the third stage, suggesting that each Epinions.com user had 6.13 trusted users. In addition, the average degree of the network increased by 0.0002 per day.

The density of the trust network changed nonmonotonically with time (Figure 11), and this change included decline and slow-growth stages. Given the lack of data on users who registered for Epinions.com before January 11, 2001, the average degree of the network relative to time was assumed to have changed sigmoidally rather than ultralinearly or nonmonotonically. However, the density of the network might have changed nonmonotonically. Based on the diffusion of innovations, Epinions.com was used at the early stages of its foundation by innovators, early adopters, and followers, who connected with each other quickly (at which point the network's density increased) and gradually discontinued their use of the website (at which point the network's density decreased). 


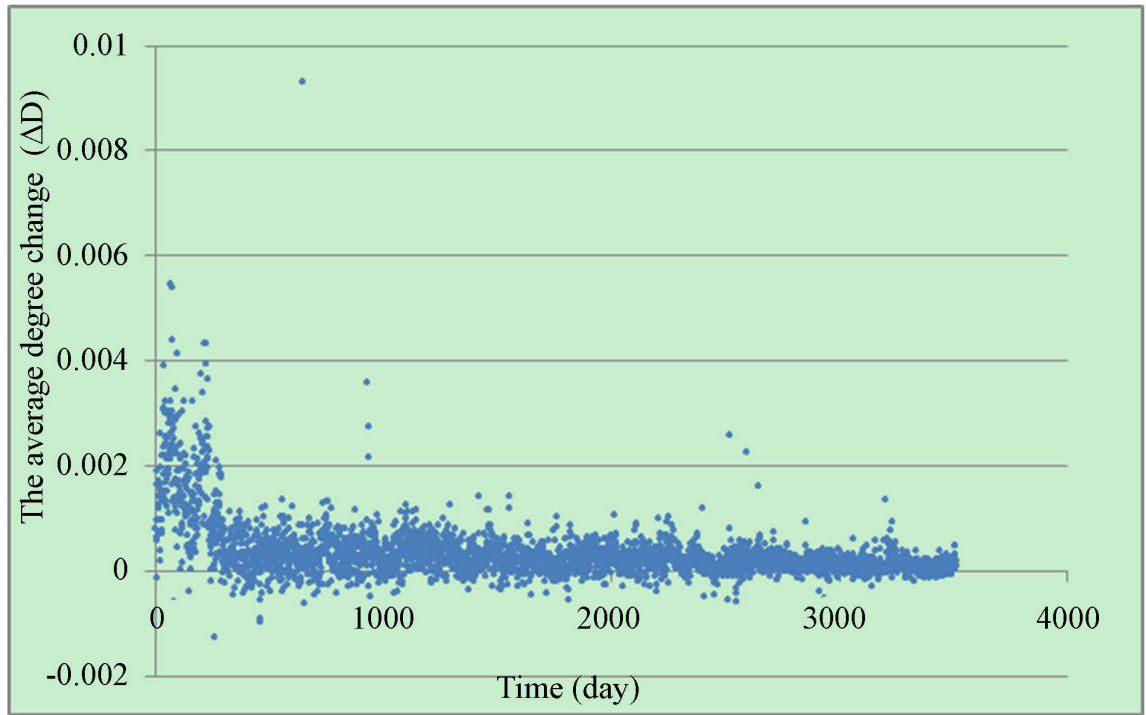

Figure 10. Change in the average degree $(\Delta D)$ of the trust network.

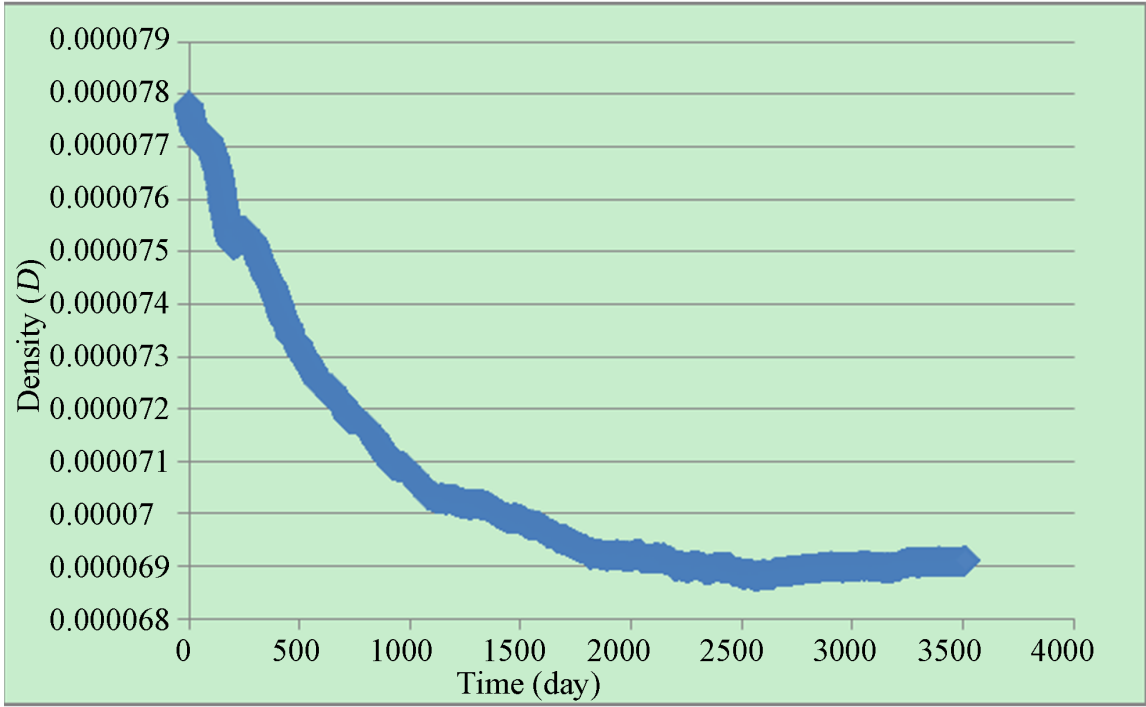

Figure 11. Density of the trust network in relation to time.

Subsequently, the website received an inflow of new users, which increased the number of edges in its network and allowed the density of its network to stabilize.

The change of the average degree of an OSN, whether ultralinearly, nonmonotonically, or sigmoidally, differs from that of a real-world social network. The average degree of a real-world social network remains constant even with the addition and elimination of nodes and edges [14].

\subsection{Growth Pattern}

\subsubsection{Growth Pattern of the Volume of Information}

Epinions.com users typically accessed the website for product reviews that helped them make purchase decisions. Thus, the volume of information on Epi- 
nions.com was defined as the number of product reviews published by the website users. The number of reviews published by the sampled 91,338 Epinions.com users was estimated, as shown in Figure 12. The growth of the total volume of information on Epinions.com was also divided into the slow stage, exponential stage, and steady stage. Compared with that of the number of network nodes and edges, the growth of information volume did not peak but grew at a faster rate at the steady stage.

\subsubsection{Growth Pattern of User Trust}

The sigmoidal growth of the network scale explained the diffusion of Epinions.com within social systems. In Epinions.com, any user who was added to another's web of trust could be perceived as having his or her review supported by that follower. The more webs of trust a user was placed in, the more followers he or she had. We therefore defined trust as a diffusion process and the growth curve of user trust as the diffusion curve of user trust to characterize the diffusion of user trust within Epinions.com. Accordingly, we assumed the trust diffusion curves of Epinions.com users to be sigmoidal.

The number of followers per day of 9247 users who had used Epinions.com for 3164 days since 2001 was estimated. Figure 13 presents the number of followers per day relative to the total number of days since registration. The number of followers per day decreased exponentially with time, corresponding with the curve of failed diffusion in the Bass model. Each of the 9347 users had only 3.72 followers, compared with an average of 6.65 followers identified in the sample population of 91,338 users. Accordingly, the trust diffusions of these 9347 users were assumed to be unsuccessful, and their trust growth curves were not bell-shaped. To validate this assumption, we selected 100 users with the highest trust levels out of the 9247 users and found that they had an average of 266.01 followers, which is considerably higher than the sample average. These users'

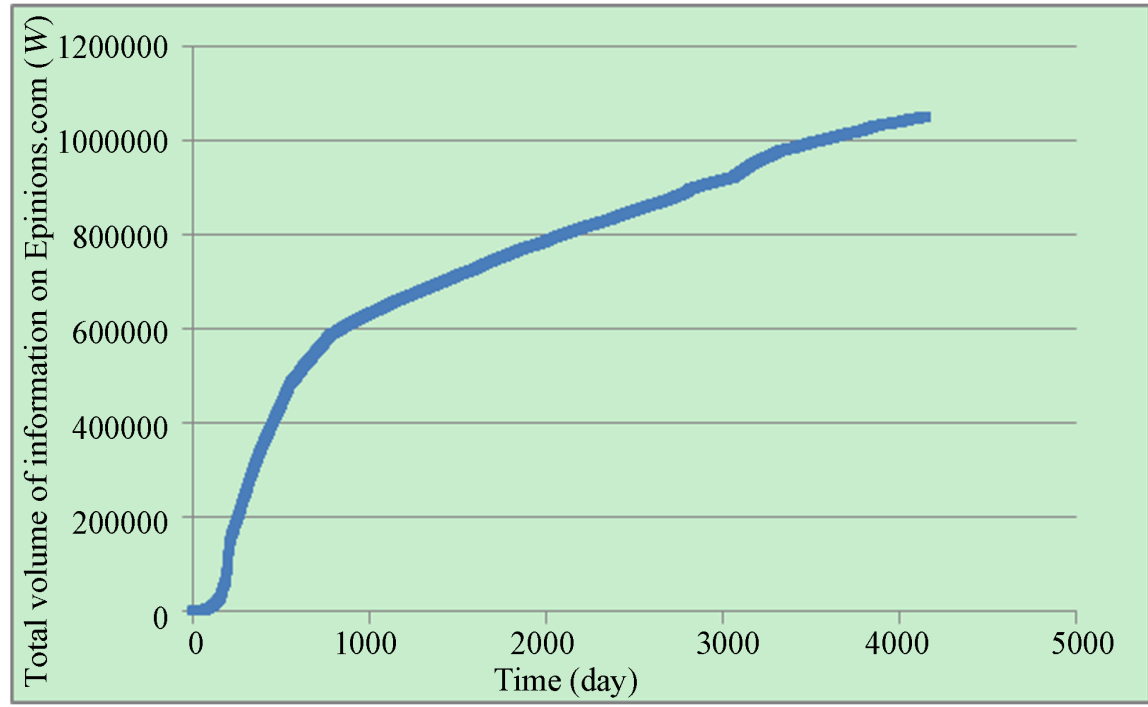

Figure 12. Cumulative growth curve of the total volume of information on Epinions.com. 
trust diffusions were perceived to be successful; their trust growth curves are plotted in Figure 14, showing peaks and bell-shaped front portions, and the axes of symmetry of the curves are located approximately at the vertical lines of the peaks. Thus, these users had successful trust diffusions on Epinions.com, and user trust diffusion in this OSN was similar to that in real-world social networks.

Trust diffusion in OSNs and real-world social networks is identically sigmoidal for two reasons. First, trust diffusion consists with diffusion of innovations, which states that the diffusion of an innovation occurs in five steps, as defined

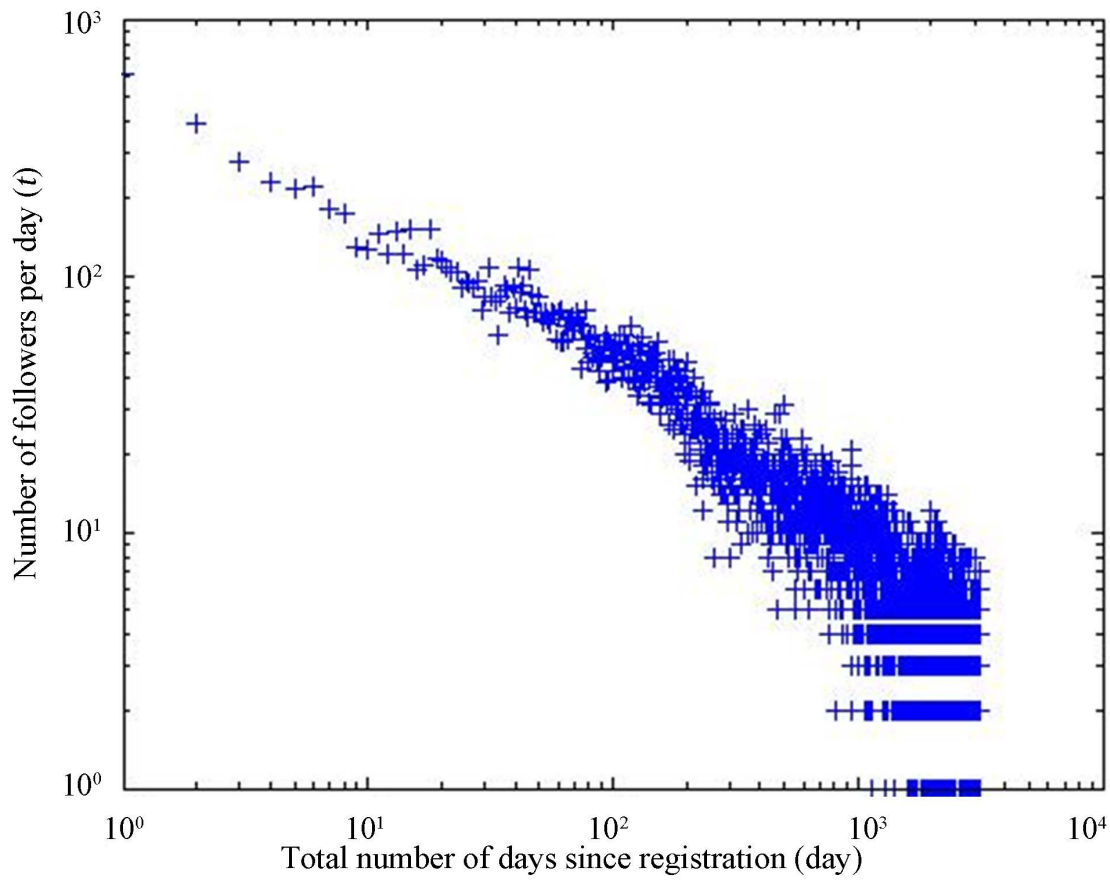

Figure 13. Growth curve of the level of user trust.

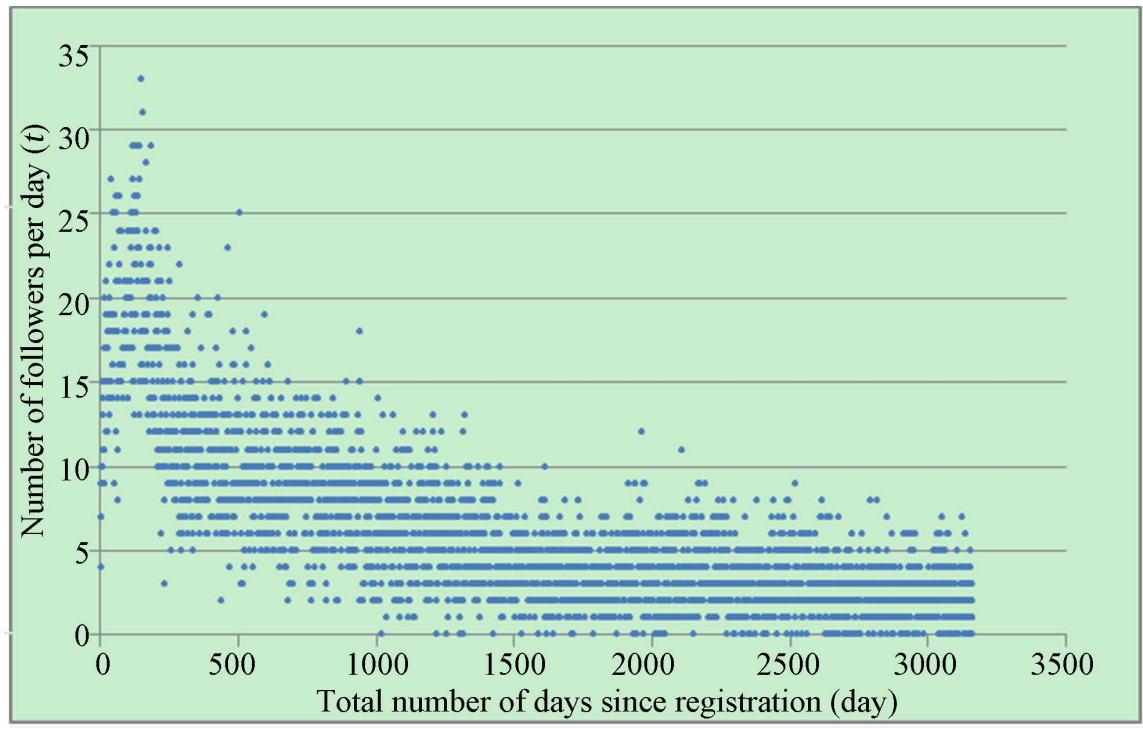

Figure 14. Growth curve of the level of user trust for the top 100 active users. 
by Rogers: awareness (a person knows the existence of an innovation but has limited knowledge of it), interest (a person starts to collect information about an innovation), evaluation (a person determines, according to his or her needs, whether to adopt an innovation), trial (a person tests an innovation to determine whether it satisfies his or her needs), and adoption (a person adopts an innovation wholeheartedly) [26]. Specifically, a user is simply aware of another user, gathers information about the user, decides whether to trust the user, evaluates the user, and makes a decision. Second, diffusion of innovations assumes mutual influence between adopters. In a trust diffusion model for real-world social networks, imitators are affected by word-of-mouth purchase recommendations. Similarly, in the trust network of Epinions.com, imitators were affected by user reputation (namely, the number of followers a user has), and if user reputation exerted a weaker effect on imitators, then user trust diffusions within the OSN exhibited higher exponentiality.

\section{Trust Model}

In this section, the pattern of trust formation in the network of Epinions.com is analyzed at the macro and micro levels.

\subsection{Macro Analysis}

Website viscosity, or "user viscosity," refers to the ability of a website to "glue" its users, which in turn can yield a direct proportion of a user's transfer cost to his or her usage, dependency, and loyalty. The higher user the viscosity a website has, the more valuable it is. Specifically, the success of social networking services depends not only on their vast number of users, but also on frequent communication between their users, which can incur high transfer costs for users. Thus, increases in the number of network edges for Epinions.com represented closer communication between its users. Its usage and viscosity also increased when edges increase.

Each increment in the number of edges in the trust network of Epinions.com represented a formation of trust on the website. Following is a macro analysis of the relationship among trust behavior, network structure, and user behavior.

We argue that the number of trust formation is relative to the OSN structure in the following four aspects. First, the number of users (or nodes) in an OSN determines the maximum number of potential trusted users that a user has in the network. Second, the number of existing edges in the network (or established trust relations) affects the formation and likelihood of repeated trust behavior. Third, participation by new users increases the number of edges (trust behavior) in the network. Fourth, trust is a type of behavior that users exhibited on Epinmions.com and could have been affected by their activeness or other types of behavior on the website.

Based on these assumptions, the network structure of Epinions.com was analyzed according to the number of existing nodes $N$, the number of existing edges 
$M$, and the increment in the number of nodes $n$ in the network; and user activeness on the website was measured according to the total number of reviews a user published, the total number of reviews rated by a user, the increment in the number of reviews a user published, and the increment in the number of reviews rated by a user. The estimation model is expressed in Equation (1):

$$
T_{t}=\beta_{1} M_{t-1}+\beta_{2} N_{t-1}+\beta_{3} W_{t-1}+\beta_{4} R_{t-1}+\beta_{5} n_{t}+\beta_{6} w_{t}+\beta_{7} r_{t}+\beta_{0}
$$

where $T_{t}$ is the increment in the number of edges at time $t\left(T_{t}=M_{t}-M_{t-1}\right)$, $M_{t-1}$ is the total number of edges at time $t-1, N_{t-1}$ is the total number of nodes at $t-1, W_{t-1}$ is the total number of reviews a user published at $t-1$, $R_{t-1}$ is the total number of reviews a user rated at $t-1, n_{t}$ is the increment in the number of nodes at $t-1\left(n_{t}=N_{t}-N_{t-1}\right), w_{t}$ is the increment in the number of reviews a user published at $t\left(w_{t}=W_{t}-W_{t-1}\right), r_{t}$ is the increment in the total number of reviews a user rated at $t\left(r_{t}=R_{t}-R_{t-1}\right)$, and $t$ is day.

SPSS was used to perform a regression analysis of Epinions.com user data from January 11 of 2001 and August 31 of 2010, the results of which are shown in Table 1.

The overall model exhibited a sufficient goodness-of-fit $\left(R^{2}=0.85\right)$ and passed the $F$ test $(F=0)$. The t-test values of all the seven variables were $\leq 0.01$, indicating a significant correlation between these variables and the increment in the number of network edges. The terms $w_{t}, r_{t}$, and $n_{t}$ were all positive, indicating the positive effects of increments in user activeness and the number of users on the formation of trust. Both $W_{t-1}$ and $R_{t-1}$ were negative, indicating the negative effects of user activeness increments on trust behavior and a diminishing returns phenomenon in the relationship between user activeness and trust behavior. The term $M_{t-1}$ was also negative, indicating that the more trust relations were established in the network, the less frequently trust behavior occurred. Finally, $N_{t-1}$ was 0 , indicating the number of users had a negligible effect on the formation of trust.

\subsection{Micro Analysis}

We also examined trust formation at the level of individual users to answer the

Table 1. Regression analysis results.

\begin{tabular}{cccc}
\hline Variable & Coefficient & $t$-stat & $\mathrm{p}$ \\
\hline$w_{t}$ & 0.174 & 12.193 & 0.00 \\
$r_{t}$ & 0.010 & 19.385 & 0.00 \\
$n_{t}$ & 2.796 & 22.270 & 0.00 \\
$M_{t-1}$ & $-4.370 \mathrm{E}-5$ & -10.141 & 0.00 \\
$W_{t-1}$ & $-3.463 \mathrm{E}-5$ & -8.578 & 0.00 \\
$R_{t-1}$ & $-3.319 \mathrm{E}-7$ & -3.468 & 0.00 \\
$N_{t-1}$ & 0.000 & 9.957 & 0.00
\end{tabular}


following questions: 1) What caused trust behavior in a user and what did not? 2) Why did only a few users have a large following, how did these users with a large following increase their trust, and what affected their trust diffusion?

\subsubsection{Hypothesis Development}

We hypothesize that the relationship between trust formation and user reputation is positive: the higher the reputation a person has, the more likely he or she is to gain trust. On Epinions.com, users with higher levels of trust (in-degree level) had higher reputation and more trust (edge connections). This is consistent with the Matthew effect, which is a fundamental hypothesis of some scalefree network models. Trust formation may also be related to user behavior. On Epinions.com, users were able to display themselves by, for example, personalizing their main pages, sharing information such as interests and hobbies, and publishing product reviews that provide insights into their experiences of using certain products or into their personalities. The more frequently users displayed themselves on the website, the more likely they were to gain trust. By contrast, users who do not display themselves in this manner might not have been noticed among their 90,000-plus counterparts; even when they did receive attention, they might have obtained limited trust because they did not make their information accessible.

Users can interact with each other, during which mutual trust occurs. For example, if User A were to perceive a review published by User B to be useful and gave a favorable response to the article, User A would therefore have had the option to follow User B, view the user's main page and other reviews (or other information related to the user), and add the user to his or her web of trust. Similarly, if User B found himself or herself followed by User A, he or she might have wanted to know about this follower and may have subsequently added the user to his or her web of trust. In summary, trust formation between users involves numerous processes, all of which are based on user behavior.

Based on our arguments, we classified the causes of trust formation into two categories: 1) user reputation and 2) user behavior and interaction. User behavior and interaction was further divided into 1) past user behavior and interaction and 2) present user behavior and interaction to examine trust formation in details, because whether trust occurred as a result of either subcategory of user behavior could not be determined. Moreover, which category of user behavior enabled a user to gain trust also could not be determined. Thus, user behavior and interaction was classified into another four subcategories: "publishing a review," "placing trust on others," "rating a review," and "receiving a review rating". Publishing a review led to user interaction only when a published review was rated. Receiving a review rating was not a type of user behavior, but it represented a type of user interaction. Placing trust on others and rating a review were user behaviors and represented user interactions. These four subcategories of user behavior were analyzed because they were widespread on Epinions.com and contained comprehensive data. Other types of frequent user behavior such 
as browsing were excluded from the analysis because no relevant data were available.

User reputation at time $t\left(D_{t}\right)$ was measured as the number of followers a user had at $t\left(\operatorname{trusted}_{t}\right)$. Thus, trusted ${ }_{t}=D_{t}-D_{t-1} \quad\left(\operatorname{trusted}_{t}\right.$ was obtained by deducting the number of followers a user had at $t-1$ from the number of followers a user had at $t$ ). The terms $T_{t}, R_{t}$, and $W_{t}$ refer to past user behavior at $t: T_{t}$ is the number of followers a user had at $t, R_{t}$ is the total number of reviews a user rated at $t$, and $W_{t}$ is the total number of reviews a user had published at $t$. The terms $t_{t}, r_{t}$, and $w_{t}$ refer to present user behavior, where $t_{t}$ is the number of trust behavior formed at $t$; thus, $t_{t}=T_{t}-T_{t-1}$ ( $t_{t}$ was obtained by deducting the number of followers at $t-1$ from the number of followers at $t$ ). The term $r_{t}$ is the total number of reviews a user rated at $t$; thus, $r_{t}=R_{t}-R_{t-1}$ ( $r_{t}$ was obtained by deducting the number of reviews a user had rated at $t-1$ from the number of reviews a user had rated at $t$ ). The term $w_{t}$ is the total number of reviews a user had published at $t$; thus, $w_{t}=W_{t}-W_{t-1}$ ( $w_{t}$ was obtained by deducting the number of reviews a user had published at $t-1$ from the number of reviews a user had published at $t$ ).

Two hypotheses were proposed, as follows:

$\mathrm{H1}$ : The number of followers a user had at $t\left(\right.$ trusted $\left._{t}\right)$ correlates positively with that user's reputation at $\mathrm{t}-1\left(D_{t-1}\right)$.

$\mathrm{H} 2$ : The number of followers a user had at $t\left(\operatorname{trusted}_{t}\right)$ correlates positively with the user's present behavior at $\mathrm{t}$ and interactions $\left(t_{t}, r_{t}, w_{t}\right.$, and $\left.r d_{t}\right)$ and with the user's past behavior at $t-1$ and past user interactions $\left(T_{t-1}, R_{t-1}, W_{t-1}\right.$, and $\left.R D_{t-1}\right)$.

\subsubsection{Model Development and Analysis}

A regression analysis was performed on panel data. After the data were subjected to a Hausma test, we adopted a fixed-effect variable intercept model. The model is expressed in Equation (2)

$$
\begin{aligned}
\operatorname{trusted}_{i t}= & a_{i}+\beta_{1} t_{i t}+\beta_{2} w_{i t}+\beta_{3} r_{i t}+\beta_{4} r d_{i t}+\beta_{5} D_{i t}+\beta_{6} T_{i t-1} \\
& +\beta_{7} W_{i t-1}+\beta_{8} R_{i t-1}+\beta_{9} R D_{i t-1}+u_{i t}
\end{aligned}
$$

where trusted $_{i t}$ the number of followers user $i$ had at $t ; t_{i t}$ is the number of formation of trust behavior user $i$ had exhibited at $t, r_{i t}$ is the number of reviews user $i$ had rated at $t, w_{i t}$ is the number of reviews that user $i$ had published at $t ; r d_{i t}$ is the number of review ratings that user $i$ had received at $t$ $D_{i t-1}$ is the reputation of user $i$ at $t-1 ; T_{i t-1}$ is the number of followers user $i$ had at $t-1 ; R_{i t-1}$ is the number of reviews user $i$ had rated at $t-1 ; W_{i t-1}$ is the number of reviews user $i$ had published at $t-1$; and $R D_{i t-1}$ is the number review ratings user $i$ had received at $t-1$.

Data on 9247 Epinions.com users that covered the period from Days 1 to 3000 since their registration in 2001 were collected. The term $t$ was defined as a 30-day cycle that started from the date of registration. The changes in the trust behavior of each of the users and in other factors affecting their behavior over 
the entire life cycle of the network since the day of registration were examined. Table 2 shows the STATA regression results.

The overall model was significant $(F=0)$ and exhibited a sufficient goodness-of-fit $\left(R^{2}=0.568\right)$. The $P$ values of all variables in the model were lower than 0.05 , indicating the significant effects of user reputation, behavior, and interaction on trust formation

Based on the coefficient results, the present user behavior and interaction positively affected the formation of the present trust behavior, indicating that the more active or interactive a user was, the more likely the user was to gain trust. The past behavior and interaction also affected the formation of the present trust behavior. Specifically, the frequencies of placing trust on others and receiving a review rating in the past positively affected the formation of present trust behavior, whereas the frequencies of publishing a review and rating a review in the past negatively affected the formation of present trust behavior.

The positive effects of placing trust on others in the past on the formation of the present trust behavior indicated that the more frequently a user placed trust on others, the more likely the user was to be trusted. This reciprocal relationship can also be observed in the real world; a person cannot be trusted without trusting others. The positive effects of receiving a rating in the past on the formation of the present trust behavior indicated that the more ratings a user received for his or her reviews (suggesting the usefulness of the reviews for other users) in the past, the more likely these reviews were to be viewed and the user to be trusted. The negative effects of publishing a review and rating a review in the past on the formation of the present trust behavior indicated diminishing returns in the positive effects of publishing a review and rating a review on the formation of the behavior. A possible explanation for this diminishing-returns phenomenon was that trust relations underlying the interactions formed between reviewers and raters allowed the formation of mostly existing interactions during review publication and rating, unless they interacted with different users to establish new trust relations.

Table 2. Estimation results.

\begin{tabular}{cccc}
\hline Variable & Coefficient & $t$-stat & $\mathrm{p}$ \\
\hline$t_{i t}$ & 0.1094825 & 373.14 & 0.00 \\
$w_{i t}$ & 0.014967 & 34.51 & 0.00 \\
$r_{i t}$ & 0.003389 & 364.70 & 0.00 \\
$r d_{i t}$ & 0.0058418 & 293.74 & 0.00 \\
$D_{i t}$ & -0.0299077 & -284.30 & 0.00 \\
$T_{i t-1}$ & 0.008875 & 129.48 & 0.00 \\
$W_{i t-1}$ & -0.0006951 & -15.34 & 0.00 \\
$R_{i t-1}$ & $-1.13 \mathrm{e}-06$ & -2.50 & 0.013 \\
$R D_{i t-1}$ & 0.0001398 & 121.82 & 0.00 \\
\hline
\end{tabular}


In addition, user reputation negatively affected the formation of the present trust behavior (Table 2), rejecting $\mathrm{H} 1$, which states that the number of followers a user had at $t\left(\right.$ trusted $\left._{t}\right)$ was positively related to user reputation at $t-1\left(D_{t-1}\right)$. This can be attributed to three factors. The first is the preferential attachment mechanism of the Barabási-Albert scale-free network model, according to which newly registered users prefer to trust users with high reputation and further trust behavior rarely occurs between longtime users and their highly reputed counterparts who were already in trust relations. The second is the limited presence of new users in a network at a state of steady expansion, which leads to a low number of new followers for highly reputed users. Thus, user reputation exerted a negative effect on the present trust formation. The third is associated with diffusion of innovations, whereby user trust diffused to only a few users of Epinions.com, despite the vast membership of the website. Thus, a user might not have been trusted by some users or, even so, added to their webs of trust. Therefore, user reputation exerted a negative effect on the number of followers because the number of potential followers decreased with the increasing number of existing followers.

In summary, factors affecting trust formation indicated the preferential attachment behavior of new edges in a trust network, suggesting that a formation of trust corresponded to an added edge in the network. These empirical findings also suggest that the preferential attachment of new edges in the OSN was based on not only the Matthew effect and the level of network degree, but also on the behavioral characteristics of nodes.

\section{Conclusions}

This study examined the static structure and dynamic evolution of the trust network of Epinions.com [33] to discuss factors that affected trust formation on this OSN.

The static structure of the trust network was illustrated in terms of out-degree, in-degree, and total-degree distributions, the shortest-path distribution, the average path length, and the average clustering coefficient. Epinions.com shared the same characteristics (namely, high clustering coefficient and short average path length) with small-world networks and exhibited a power-law degree distribution, rendering it a scale-free network.

An analysis of the dynamic evolution of the trust network yielded the following findings. The cumulative growth in the scale of the network was sigmoidal, corresponding with the Bass model. The average degree of the network was nonconstant, compared with the constant one of a real-world network. The average degree of the network changed into a bell-shaped curve, indicating that the cumulative growth curve of the average degree of the network in its entire life was also sigmoidal. The density of the network decreased and subsequently stabilized, indicating that the network density increased at the early stage of the network, when trust relations were rapidly established by aficionados. 
Trust diffusion in the network varied from user to user. Trust diffusion failed for most of the sampled users, corresponding to the curve of failed diffusion in the Bass model. The growth curve of the level of user trust was bell-shaped for users with successful trust diffusion.

Trust formation was examined at the macro- and micro-levels. In terms of the overall network (macro-level), trust formation was related to user activeness and the structure of the trust network in which users participated. Specifically, the number of formation of present trust behavior correlated positively with user activeness and the number of newly registered users, although user activeness exerted diminishing-returns effects on trust formation. In addition, existing trust relations negatively affected newly established ones, and the number of users slightly affected trust formation. In terms of individual users (micro-level), trust formation was related to user reputation, behavior, and interaction. The formation of the present trust behavior was negatively affected by user reputation but positively affected by present user behavior and interaction; review publication and rating had diminishing-returns effects on trust formation; and trust behavior and the number of reviews rated had cumulative effects on trust formation.

In summary, trust formation in OSNs depends more on a user activeness than his or her accumulated contribution and reputation. Accordingly, diminishing returns exist in the relationship between user activeness and trust formation in OSNs, in contrast to the Matthew effect in real-world social networks.

\section{Acknowledgements}

This work was supported, in part, by the National Natural Science Foundation of China (Grant Nos. 70801066, 71071167, 71071168, 71371200), and by a grant from Sun Yat-sen University Basic Research Funding (Grant Nos. 1009028, 1109115, 16wkjc13).

\section{References}

[1] Adamic, L.A., Buyukkokten, O. and Adar, E. (2003) A Social Network Caught in the Web. First Monday, 8. https://doi.org/10.5210/fm.v8i6.1057

[2] Shi, X., Adamic, L.A. and Strauss, M.J. (2006) Networks of Strong Ties. Physica $A$ Statistical Mechanics \& Its Applications, 378, 33-47. https://doi.org/10.1016/j.physa.2006.11.072

[3] Golder, S.A., Wilkinson, D.M. and Huberman, B.A. (2007) Rhythms of Social Interaction: Messaging within a Massive Online Network. Springer, London.

[4] Feng, F., Chen, X., Liu, L. and Long, W. (2007) Social Dilemmas in an Online Social Network: The Structure and Evolution of Cooperation. Physics Letters A, 371, 58-64. https://doi.org/10.1016/j.physleta.2007.05.116

[5] Ahn, Y.Y., Han, S., Kwak, H., Moon, S. and Jeong, H. (2007) Analysis of Topological Characteristics of Huge Online Social Networking Services. Proceedings of the 16th International Conference on World Wide Web, Alberta, 8-12 May 2007, 835-844. https://doi.org/10.1145/1242572.1242685

[6] Wakita, K. and Tsurumi, T. (2007) Finding Community Structure in Mega-Scale Social Networks. Proceedings of the 16th International Conference on World Wide 
Web, Alberta, 8-12 May 2007, 1275-1276. https://doi.org/10.1145/1242572.1242805

[7] Yuta, K., Ono, N. and Fujiwara, Y. (2007) A Gap in the Community-Size Distribution of a Large-Scale Social Networking Site. Arxiv:/Physics/0701168, Arxiv.Org

[8] Csányi, G. and Szendroi, B. (2003) Structure of a Large Social Network. Physical Review E Statistical Nonlinear \& Soft Matter Physics, 69, Article ID: 036131. https://doi.org/10.1103/PhysRevE.69.036131

[9] Ghoshal, G. and Holme, P. (2005) Attractiveness and Activity in Internet Communities. Physica A Statistical Mechanics \& Its Applications, 364, 603-609. https://doi.org/10.1016/j.physa.2005.04.047

[10] Holme, P., Edling, C.R. and Liljeros, F. (2004) Structure and Time Evolution of an Internet Dating Community. Social Networks, 26, 155-174. https://doi.org/10.1016/j.socnet.2004.01.007

[11] Holme, P. (2003) Network Dynamics of Ongoing Social Relationships. Europhysics Letters, 64, 427-433. https://doi.org/10.1209/epl/i2003-00505-4

[12] Adamic, L. and Adar, E. (2003) How to Search a Social Network. Social Networks, 27, 187-203. https://doi.org/10.1016/j.socnet.2005.01.007

[13] Adamic, L.A. and Adar, E. (2003) Friends and Neighbors on the Web. Social Networks, 25, 211-230. https://doi.org/10.1016/S0378-8733(03)00009-1

[14] Kumar, R., Novak, J. and Tomkins, A. (2006) Structure and Evolution of Online Social Networks. Proceedings of the 11 th ACM SIGKDD International Conference on Knowledge Discovery and Data Mining, Chicago, 21-24 August 2006, 611-617. https://doi.org/10.1145/1150402.1150476

[15] Zakharov, P. (2006) Diffusion Approach for Community Discovering within the Complex Networks: Live Journal Study. Physica A Statistical Mechanics \& Its Applications, 378, 550-560. https://doi.org/10.1016/j.physa.2006.11.086

[16] Goh, K.I., Eom, Y.H., Jeong, H., Kahng, B. and Kim, D. (2006) Structure and Evolution of Online Social Relationships: Heterogeneity in Unrestricted Discussions. Physical Review E Statistical Nonlinear \& Soft Matter Physics, 73, Article ID: 066123. https://doi.org/10.1103/PhysRevE.73.066123

[17] Wang, F., Moreno, Y. and Sun, Y. (2006) Structure of Peer-to-Peer Social Networks. Physical Review E Statistical Nonlinear \& Soft Matter Physics, 73, Article ID: 036123. https://doi.org/10.1103/PhysRevE.73.036123

[18] Leskovec, J. and Horvitz, E. (2008) Planetary-Scale Views on a Large Instant-Messaging Network. Proceedings of the 17 th International Conference on World Wide Web, Beijing, 21-25 April 2008, 915-924.

[19] Leskovec, J., Backstrom, L., Kumar, R. and Tomkins, A. (2008) Microscopic Evolution of Social Networks. Proceedings of the 14 th ACM SIGKDD International Conference on Knowledge Discovery and Data Mining, Las Vegas, 24-27 August 2008, 462-470. https://doi.org/10.1145/1401890.1401948

[20] Smith, R.D. (2002) Instant Messaging as a Scale-Free Network.

[21] Zhang, J., Ackerman, M.S. and Adamic, L. (2007) Expertise Networks in Online Communities: Structure and Algorithms. Proceedings of the 16th International Conference on World Wide Web, Alberta, 8-12 May 2007, 221-230. https://doi.org/10.1145/1242572.1242603

[22] Mislove, A., Koppula, H.S., Gummadi, K.P., Druschel, P. and Bhattacharjee, B. (2008) Growth of the Flickr Social Network. Proceedings of the First Workshop on Online Social Networks, Seattle, 18 August 2008, 25-30.

https://doi.org/10.1145/1397735.1397742 
[23] Chun, H., Ahn, Y.Y., Kwak, H., Moon, S., Eom, Y.H. and Jeong, H. (2008) Comparison of Online Social Relations in Terms of Volume vs. Interaction. Catalysis Today, 36, 431-439.

[24] Torkjazi, M., Rejaie, R. and Willinger, W. (2009) Hot Today, Gone Tomorrow: On the Migration of MySpace Users. Proceedings of the 2nd ACM Workshop on Online Social Networks, Barcelona, 17 August 2009, 43-48. https://doi.org/10.1145/1592665.1592676

[25] Hu, H.B. (2010) Research on the Structure, Evolution and Dynamics of Online Social Networks. PhD Thesis, Shanghai Jiao Tong University, Shanghai.

[26] Rogers, E. (2002) Diffusion of Innovations. 4th Edition, the Central Compilation and Translation Press, Beijing.

[27] Bass, F.M. (1969) A New Product Growth for Model Consumer Durables. Management Science, 15, 215-227. https://doi.org/10.1287/mnsc.15.5.215

[28] Bai, S.Y. (2003) The Formation Mechanisms of Online Interpersonal Trust. Academic Exchange, 3, 95-97.

[29] Huang, H.M. (2001) Identity and Trust in Virtual Communities. PhD Thesis, National Taiwan University, Taipei.

[30] Milgram, S. (1967) The Small World Problem. Psychology Today, 2, 185-195.

[31] Bates, D.M. and Watts, D.G. (1988) Nonlinear Regression Analysis and Its Applications. Wiley, New York. https://doi.org/10.1002/9780470316757

[32] Xu, X., Hu, J. and Liu, F. (2007) Complex Network Study of Asian Go Players. Chaos, 17, Article ID: 023111. https://doi.org/10.1063/1.2722040

[33] Li, Y., Cao, H.D., Zhang, Y. and Li, B.B. (2018) Characteristics of Human Behavior in an Online Society. SageOpen, 1-13. https://doi.org/10.1177/2158244018770494 